\title{
Improvement of Congenital Muscular Torticollis with Mild Symptoms in Non-Treated Adult after Simple Surgical Myotomy of Sternocleidomastoid Muscle under Local Anesthesia
}

Young Hoo Joh, Dong Ha Park,

II Jae Lee, Myong Chul Park

Department of Plastic and Reconstructive Surgery, Ajou University School of Medicine, Suwon, Korea

No potential conflict of interest relevant to this article was reported.

\begin{abstract}
In adult congenital muscular torticollis (CMT) patients, physical therapy is not as effective because the development of sternocleidomastoid muscle (SCM) muscle is complete. While surgical release can address CMT in adult patients, the risk of general anesthesia and visible postoperative scar is a concern, expecially in patients with mild symptoms. In this paper, we report our experience in treating such patients with minimal-incision myotomy under local anesthesia. A review was performed for all adult patients who had undergone the simple myotomy procedure. Surgical indication was reserved for patients with mild fibrotic band in the SCM muscle with minimal lengthdiscrepancybetween the muscles. All patients had recognizable head tiltand palpation of fibrotic band on affected side of the neck. Surgical details are described in the main body of text. Three female patients had undergone the procedure. Torticollis was resolve in all patients with complete restoration of ranage of motion. There were no postoperative complications, and patient satisfaction was high. We have reported three cases of mild CMT in adult female patients, who had undergone minimal-incision myotomy under local anesthesia. Outcomes were satisafactory with no morbidity to report. With careful patient selection, this method offers an alternate treatment option for adult CMT patients with mild symptoms.
\end{abstract}

Keywords: Congenital torticollis / Surgical procedures / Local anesthesia

\section{INTRODUCTION}

Congenital muscular torticollis (CMT) is caused by an idiopathic fibromatous contracture of the sternocleidomastoid muscle (SCM). This contracture will cause the head to turn towards the affected side, with the chin rotates to the contralateral side. If left unrecognized and untreated, CMT can result in facial asymme-

Correspondence: Myong Chul Park

Department of Plastic and Reconstructive Surgery, Ajou University School of Medicine, 164 World cup-ro, Yeongtong-gu, Suwon 16499, Korea

E-mail: mpark@ajou.ac.kr

Received May 9, 2015 / Revised June 3, 2015 / Accepted August 4, 2015 try, abnormal spine growth, and plagiocephaly $[1,2]$.

Operative intervention is indicated for patients who do not respond to rehabilitation or in whom the fibrosis is severe [3,4]. Many studies have shown that surgical release of fibrosis can normalize the head tilt in children. However, treatment algorithm is not so clear for CMT in adult with mild symptoms and minimal fibrosis.

In adult CMT patients, physical therapy is not as effective because the development of SCM muscle is complete. While surgical release can address CMT in adult patients, the risk of general anesthesia and visible postoperative scar is a concern, expecially in patients with mild symptoms. In this paper, we report our ex-

88 Copyright @ 2015 The Korean Cleft Palate-Craniofacial Association 
perience in treating such patients with minimal incision myotomy under local anesthesia.

\section{CASE REPORT}

Three female patients had undergone minimal incision myotomy under local anesthesia (Table 1). All of the patients had mildly tilted head and palpable fibrotic band to the affected side, which the patients themselves had noticed since early childhood but never sought medical attention prior to visiting our clinic. Two patients had subjective flexion difficulty with clinical flexion deficit was less than 15 degrees. The third patient did not complain of limited range of motion. Mild fibrosis of SCM muscle was confirmed on physical examination and magnetic resonance imaging (MRI) in all patients. Ultrasonography was used for precise localization of fibrotic portion of the muscle, which provided useful target for surgical release in two patients (Fig. 1).

In the operating room and anesthesiologist present, the fibrotic portion was palpated and correlated with available ultrasonographic information. The area of concern was infiltrated with a dilution of 2\% lidocaine and1:100,000 epinephrine. A sub-centimeter incision was made through the dermis, and blunt dissection was carried past the platysma down to the SCM muscle. The head was rotated to the contralateral side to minimize the risk of injury to critical structures deep to the muscle. Under direct visualization, the fibrotic portion of the SCM was meticulously transected using electrocautery (Fig. 2). During this process, the

Table 1. Summary of mild congenital muscular torticollis cases

\begin{tabular}{|c|c|c|c|c|c|}
\hline \multirow[t]{2}{*}{$\begin{array}{l}\text { Sex } \\
\text { Age } \\
\text { (yr) }\end{array}$} & \multirow[t]{2}{*}{$\begin{array}{l}\text { Affected } \\
\text { side }\end{array}$} & \multirow[t]{2}{*}{ Symptoms } & \multirow{2}{*}{$\begin{array}{l}\text { Follow- } \\
\text { up } \\
\text { periods } \\
\text { (mo) }\end{array}$} & \multicolumn{2}{|c|}{$\begin{array}{l}\text { Satisfaction } \\
\text { after surgery } \\
(\text { scale } 1-5)^{a)}\end{array}$} \\
\hline & & & & $\begin{array}{l}\text { Relief of } \\
\text { symptoms }\end{array}$ & Scar \\
\hline $\mathrm{F} / 18$ & Right & $\begin{array}{l}\text { 1. Tilted head } \\
\text { 2. Fibrotic band palpation }\end{array}$ & 13 & 5 & 5 \\
\hline$F / 30$ & Left & $\begin{array}{l}\text { 1. Tilted head } \\
\text { 2. Fibrotic band palpation } \\
\text { 3. Subjective flexion difficulty }\end{array}$ & 13 & 4 & 5 \\
\hline$F / 31$ & Right & $\begin{array}{l}\text { 1. Tilted head } \\
\text { 2. Fibrotic band palpation } \\
\text { 3. Subjective flexion difficulty }\end{array}$ & 15 & 5 & 5 \\
\hline
\end{tabular}

a) 5 , very good; 4 , good; 3 , satisfactory; 2 , poor; 1 , very poor. hard fibrotic band was pinched to immobilize the SCM and to further minimize the risk of inadvertent injury to underlying structures. Surgical release was confirmed intraoperatively by testing passive range of motion prior to closure of the wound.

There were no postoperative complications to report. All of the patients demonstrated resolution of head tilt and had full range of motion (Fig. 3). All of the patients continued to be satisfied with treatment outcomes at 12-month follow up and did not report recurrence of symptoms.

\section{DISCUSSION}

Adult form of CMT is considered to be relatively rare in western literature. In South Korea, however, CMT was not recognized as a clinically treatable condition to the public until the past decade. Because of this, many of the parents did not have the opportunities to seek medical attention for children with CMT, and adult
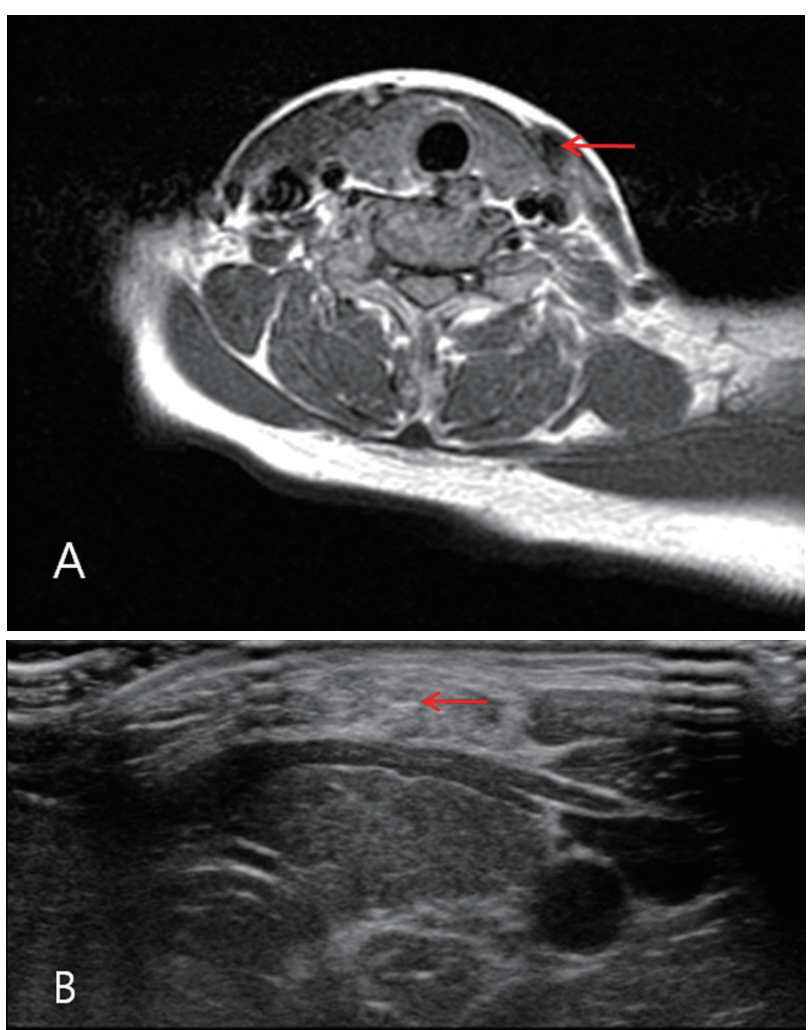

Fig. 1. Preoperative imaging studies confirms mild fibrosis (red arrows) in the sternocleidomastoid muscle. (A) Magnetic resonance imaging. (B) Ultrasonography. 
CMT may not be as rare in this country for this reason. This appears to be especially true for children with milder forms of CMT.

Severely fibrotic and contracted SCM muscle provides a relatively good indication for operative intervention regardless of age. Surgical release of the fibrosis returns the sagittal plane of the closer to the spinal axis $[5,6]$, with many variations in surgical techniques [4-8]. At our department, severe SCM fibrosis is accessed via a supraclavicular incision. The fibrotic band is transected, and this results in drastic improvement of torticollis with virtually nil complication among hundreds of cases [3,9]. Despite the outcome, the operation must weight the benefits of operation against the potential risk from general anesthesia, posteropative scar, and immobilization of neck until 2 weeks from operation date.
Mild forms of CMT can be treated with physical therapy in infants and children [10]. While a child is still growing, rehabilitation can lengthen the contracted SCM muscle. If rehabilitation does not adequately improve the condition after a protracted period of treatment, surgical intervention becomes an option.

The gray area of management in CMT is those adults with mild forms of CMT. In adult patients, rehabilitation does not work, as the SCM is no longer under developmental influence. In these patients, the risk of general anesthesia and conspicuous scar seems to outweigh the improvement to be gained from treating the mild CMT.

The minimal-incision technique described in this current study was developed to address these mild forms of CMT in adult patients. Because of this, we restrict the indication to patients with
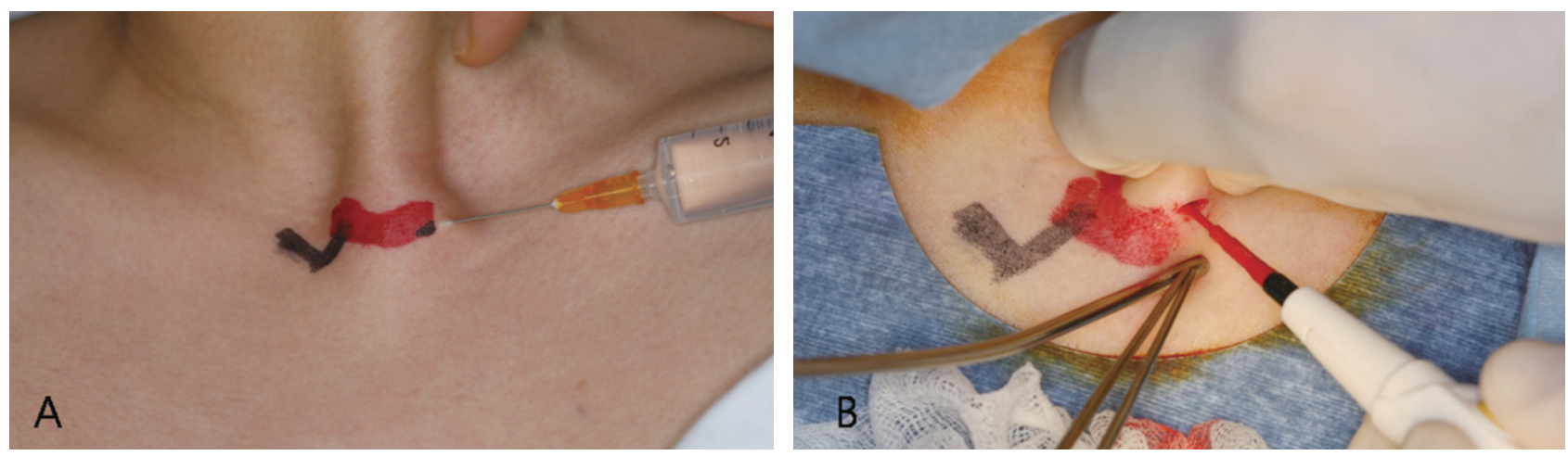

Fig. 2. Intraoperative photographs of simple myotomy. (A) In the operating room and anesthesiologist present, the fibrotic portion was palpated and correlated with available ultrasonographic information. The area of concern was infiltrated with a dilution of 2\% lidocaine and 1:100,000 epinephrine. (B) The sternocleidomastoid muscle is pinched during electrocauterization of the fibrotic tissue in order to avoid injury to deeper structures.
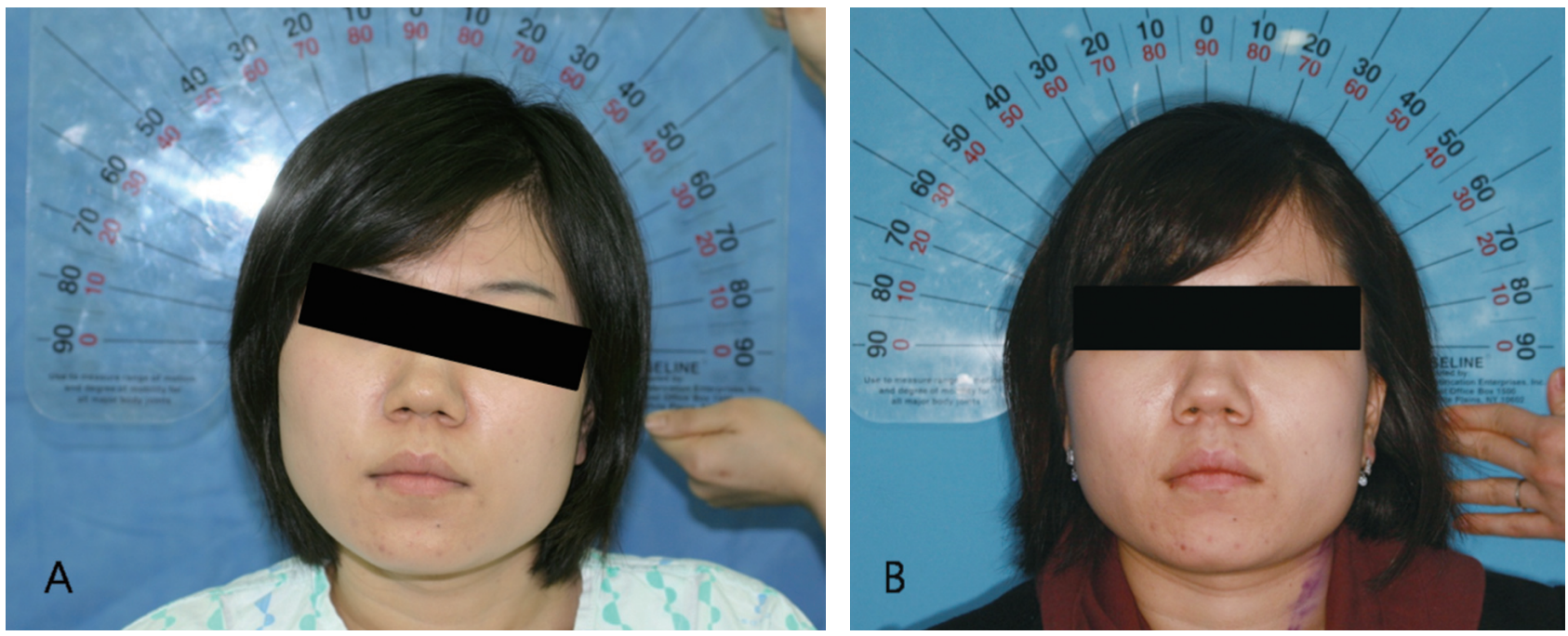

Fig. 3. A 30-year-old female patient with mild muscular torticollis of the left sternocleidomastoid muscle before (A) and after (B) the simple myotomy. 
minimal length discrepancy between bilateral SCM muscle, minor fibrotic band, and neck flexion disability less than 15 degrees of deficit. The SCM muscles were evaluated with preoperative MR imaging. For the approximating the location of fibrosis, ultrasonography was used for identification of proper incision line and intraoperative approach. The authors feel strongly that this procedure should be performed by surgeons who are extremely familiar with the head and neck anatomy and have performed open exploration more than on a handful of occasions, as inadvertent injury to the deeper critical structures is unacceptable in the context of patients with relatively minor functional issue. A full transection of the fibrotic band is the principal goal of myotomy. Complete release is confirmed with increased and total permission of passive range of motion.

All patients were female patients, who did not wish to have a large conspicuous scar. We feel that postoperative satisfactions were high because the tradeoff between minimal scar and resolution of CMT was acceptable to these patients.

This minimal-incision myotomy technique is extremely inappropriate in patients with moderate-to-severe torticollis and/or extensive fibrosis of SCM. In suitable surgical candidate, the simple myotomy method lead to appropriate lengthening effect of affected SCM muscle and release of fibrotic band with no visible scar. Strict physical therapy is still required to prevent re-adhesion and contracture. The patients wore Thomas-type cervical collar for one week following the operation. After the first week, patients were instructed on neck exercise with contralateral flexion and ipsilateral rotation. All of the patients were found to have full range of motion of neck and improvement in cervical posture at the last clinic follow up.

Open SCM muscle surgery is the conventional treatment for torticollis, with favorable outcomes [11]. In our study, we have reported three cases of mild CMT in adult female patients, who had undergone minimal-incision myotomy under local anesthesia. Outcomes were satisafactory with no morbidity to report. With careful patient selection, this method offers an alternate treatment option for adult CMT patients with mild symptoms.

\section{REFERENCES}

1. Yu CC, Wong FH, Lo LJ, Chen YR. Craniofacial deformity in patients with uncorrected congenital muscular torticollis: an assessment from three-dimensional computed tomography imaging. Plast Reconstr Surg 2004;113:24-33.

2. Hollier L, Kim J, Grayson BH, McCarthy JG. Congenital muscular torticollis and the associated craniofacial changes. Plast Reconstr Surg 2000;105:827-35.

3. Lee IJ, Lim SY, Song HS, Park MC. Complete tight fibrous band release and resection in congenital muscular torticollis. J Plast Reconstr Aesthet Surg 2010;63:947-53.

4. Cheng JC, Tang SP. Outcome of surgical treatment of congenital muscular torticollis. Clin Orthop Relat Res 1999;(362):190-200.

5. Bharadwaj VK. Sternomastoid myoplasty: surgical correction of congenital torticollis. JOtolaryngol 1997;26:44-8.

6. Shim JS, Jang HP. Operative treatment of congenital torticollis. J Bone Joint Surg Br 2008;90:934-9.

7. Wirth CJ, Hagena FW, Wuelker N, Siebert WE. Biterminal tenotomy for the treatment of congenital muscular torticollis. Long-term results. J Bone Joint Surg Am 1992;74:427-34.

8. Stassen LF, Kerawala CJ. New surgical technique for the correction of congenital muscular torticollis (wry neck). Br J Oral Maxillofac Surg 2000;38:142-7.

9. Seo SJ, Yim SY, Lee IJ, Han DH, Kim CS, Lim H, et al. Is craniofacial asymmetry progressive in untreated congenital muscular torticollis? Plast Reconstr Surg 2013;132:407-13.

10. Cheng JC, Wong MW, Tang SP, Chen TM, Shum SL, Wong EM. Clinical determinants of the outcome of manual stretching in the treatment of congenital muscular torticollis in infants: a prospective study of eight hundred and twenty-one cases. J Bone Joint Surg Am 2001;83:679-87.

11. Ippolito E, Tudisco C, Massobrio M. Long-term results of open sternocleidomastoid tenotomy for idiopathic muscular torticollis. J Bone Joint Surg Am 1985;67:30-8. 